\title{
DiNÁMICA DE COINTEGRACIÓN EN LA PARIDAD IMPLÍCITA DEL EURodólar en la RePÚblica Dominicana
}

\author{
EnRique Penson ${ }^{a}$
}

Recibido: marzo, 2019 • Aceptado: julio, 2019

Cómo citar: Penson, E. (2019). Dinámica de cointegración en la paridad implícita del eurodólar en la República Dominicana. Ciencia, Economía y Negocios, 3(1), 87-106. Doi: https://doi.org/ 10.22206/ceyn.2019.v3i1.pp87-106

\section{Resumen}

El presente estudio explora la relación entre la razón del tipo de cambio local pesoleuro y pesoldólar, efectivamente una tasa eurodólar implícita, con el eurodólar oficial internacional. Se encontró que el eurodólar implícito local mantiene una relación de equilibrio de largo plazo con su contraparte internacional; cuando existieron desajustes, la velocidad de convergencia promedio se encontraba alrededor de cinco días previo a la implementación del esquema de metas de inflación del Banco Central, mas ahora pasó a ser de alrededor 20 dias. Estos resultados reflejan tres condiciones importantes del mercado financiero dominicano actual: primero, existe un vinculo estrecho entre el desenvolvimiento de los mercados globales y el mercado local; segundo, aún existen oportunidades de arbitraje en el corto plazo en el mercado de divisas; y tercero, que la implementación de un régimen de metas de inflación ha tenido un costo en República Dominicana que parece reflejarse a través de cierta rigidez en el mercado cambiario.

Palabras clave: cointegración; modelo de corrección de errores; mercado cambiario; metas de inflación.

Códigos JEL: F31, F41, G15, E42.

aAnalytica. Dirección: Avenida Sarasota No. 29, Mirador Sur, Santo Domingo, República Dominicana. T: +18098857775, correo-e: e.penson.brisindi@gmail.com

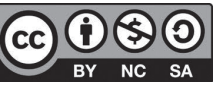




\title{
COINTEGRATION DYNAMICS IN THE IMPLICIT EURODOLLAR PARITY IN THE DOMINICAN REPUBLIC
}

\author{
Enrique Penson
}

Received: March, 2019• Accepted: July, 2019

\begin{abstract}
This study explores the relationship between the ratio between local pesoleuro and pesoldollar exchange rate, effectively an implicit Eurodollar rate, with the official international Eurodollar rate. One finds that the implicit local Eurodollar rate does maintain an equilibrium relationship with its international counterpart; furthermore, when maladjustments occur, the average velocity at which the local rate converges to equilibrium was around five days prior to the inflation targeting regime implementation, and now sits at around 20 days. These results reflect three important conditions in the current Dominican financial market: First, there exists a tight relationship between global market developments and the local market. Second, some arbitrage opportunities still occur in the local exchange rate market. Third, and final, the implementation of an inflation targeting regime appears to have borne a cost in the way of a certain measure of rigidity in the exchange rate market.
\end{abstract}

Keywords: Cointegration; error correction model; exchange rate market; inflation targets.

JEL Codes: F31, F41, G15, E42. 


\section{Introducción}

El estudio de la dinámica de los tipos de cambio en República Dominicana tradicionalmente se ha enfocado en la relación entre el peso dominicano y el dólar estadounidense. Este enfoque de la literatura dominicana en la razón peso-dólar, acertadamente obedece a la importancia de las relaciones comerciales entre República Dominicana y Estados Unidos, pero deja al lado la otra razón cambiaria más importante en el sistema financiero dominicano, el tipo de cambio peso/euro.

Tan importante es el mercado de divisas peso-dólar en relación al peso-euro, que solo existe información públicamente diseminada por el Banco Central sobre el volumen de transacciones del primero, y no del segundo; sin embargo, es posible contar con una indicación del diferencial de tamaño entre ambos al observar que, al cierre de 2018, los depósitos en dólares estadounidenses equivalían a $27.2 \%$ del total de depósitos en el sistema financiero consolidado de República Dominicana, mientras que los depósitos en euros solo ascendían a $0.6 \%$ del total. Lo pequeño que es el mercado de euros en el país, si se toman los depósitos como una indicación, no lo hace menos importante, ya que su interacción con el mercado de dólares tiene la capacidad de brindar luz sobre las dinámicas subyacentes de ambos mercados.

En la medida que el sistema financiero dominicano tiene cierto nivel de profundidad, es de esperar que los tipos de cambio peso/dólar y peso/ euro reflejen el tipo de cambio eurodólar internacional; de no respetarse este equilibrio en el largo plazo, sería posible aprovechar un desequilibrio de mercado para lograr ganancias extraordinarias por arbitraje. El presente estudio trata exactamente de discernir la presencia de este fenómeno.

$\mathrm{Al}$ respecto, cabe preguntarse: ¿la razón cambiaria peso/dólar y peso/ euro en el sistema financiero dominicano se encuentra en equilibrio con el tipo de cambio eurodólar en el mercado internacional? ¿Existen desequilibrios en esta relación? ¿Cuántos días toma el mercado para retornar a su equilibrio de largo plazo? ¿Cambió esta dinámica posterior a la implementación del esquema de metas de inflación por parte del Banco Central de la República Dominicana? 
Este trabajo responde las preguntas anteriores a través de la implementación de un modelo de corrección de errores (ECM, por sus siglas en inglés), que logra estimar tanto la relación de largo plazo o equilibrio entre el tipo de cambio implícito eurodólar local y el internacional como estimar la dinámica de corto plazo y su velocidad de corrección al largo plazo. Los resultados de este análisis apuntan a que sí existe una relación de equilibrio de largo plazo hacia la que la razón peso/dólar vs peso/euro local se corrige en el corto plazo. Se encuentra que la velocidad promedio de corrección de largo plazo era de cinco días previo a la implementación del esquema de metas de inflación, posterior al cual pasó a ser alrededor de 20 días.

\section{Revisión de la literatura}

Para los fines del presente estudio es oportuno explorar tanto las experiencias internacionales en materia del funcionamiento del mercado de divisas y el arbitraje como el uso de técnicas de cointegración para medir el funcionamiento de estos mercados y la posibilidad de arbitraje, sumado a los trabajos que han tratado de indagar sobre los precios del mercado de divisas en República Dominicana.

\section{Arbitraje y mercados de divisas internacionales}

La ley de un precio, uno de los axiomas fundamentales de la disciplina económica, afirma que cualquier bien puede tener un solo precio (Rashid, 2007); esto significa que, por ejemplo, luego de descontar diferencias en costos de transacciones, dos barriles de petróleo idénticos deben transarse por el mismo precio siempre, que no debe ser posible financiarse en una moneda para invertir en otra con un retorno positivo seguro, y que las razones cambiarias entre monedas deben mantener coherencia entre sí.

La paradoja del arbitraje, según notaran por primera vez Grossman y Stiglitz (1976 y 1980), radica en que, si el arbitraje no ocurre, entonces los participantes de un mercado no tienen incentivos para buscar su evidencia, y por tanto podrían aparecer oportunidades de arbitraje. Esta evidencia de arbitraje en los diferentes mercados existe, y está referida 
en revisiones de autores como Akram, Rime y Sarno (2008), quienes encuentran que existen oportunidades de arbitraje en los mercados de capitales que facilitan la realización de ganancias extraordinarias, pero que su duración es limitada.

Adicionalmente, existe evidencia de que los hacedores de política monetaria, al ejecutar su mandato de estabilización de precios, a veces distorsionan las relaciones entre los precios de mercado, según establecen McCallum (1994); Baillie y Osterberg (2000); Chinn y Meredith (2004); y Mark y Moh (2007).

\section{Uso de técnicas de cointegración en evaluación de mercados de divisas}

La cointegración, propuesta originalmente por Granger y Newbold (1974), es una propiedad estadística de las series de tiempo en que existen casos de dos o más series que mantienen cierta relación entre sí y que es relativamente estable.

El ejercicio de realizar pruebas empíricas que presenten evidencia sobre cointegración entre cualesquiera precios de activos financieros (como las divisas) es, por definición, una violación de la tesis de los mercados eficientes de Fama (1970), ya que, de probarse cointegración, una de las series cointegradas puede ser predicha, indicando que el mercado no ha incorporado esa información aún (contrario a lo que la teoría apunta), según lo indicado por Granger (1986).

A pesar de que la teoría financiera de los mercados eficientes no incorpora esta posibilidad de precios cointegrados, la literatura sobre los mercados de divisas que rechaza la hipótesis de no cointegración es vasta. Algunos de los trabajos que encuentran evidencia de no cointegración son Baillie y Bollerslev (1989, 1994); Copeland (1991); Sephton y Larsen (1991); Diebold, Gardeazabal, y Yilmaz (1994); Norrbin (1994); Lajaunie y Naka (1997); Woo (1999); Haug, MacKinnon, y Michelis. (2000). Adicionalmente, la literatura ha encontrado que la presencia o no de cointegración tiene cierto grado de sensibilidad al rango de tiempo utilizado para probarla (Sephton y Larsen, 1991; Phengpis, 2006). 


\section{República Dominicana y el estudio del tipo de cambio}

La literatura sobre el tipo de cambio en la República Dominicana tradicionalmente se ha enfocado en la razón cambiaria entre el peso dominicano y el dólar estadounidense (DOP/USD). Este tipo de cambio peso-dólar ha podido ser entendido usando modelos de macroeconomía para economías abiertas.

Los trabajos de Sánchez-Fung (1999, 2014) y Andújar y Medina (2008) han mostrado que la razón cambiaria peso-dólar puede ser explicada en función de la paridad del poder adquisitivo (incorporando el nivel de precios estadounidense y el dominicano al análisis), y también introduciendo aspectos microeconómicos como la compra y venta de divisas en el mercado local.

Posteriores trabajos como Bello, Heresi y Pineda (2010), Vásquez-Ruíz y Rivas (2012, 2014), Gratereaux (2015) y Cruz (2016) confirman esta tesis con datos más actualizados, incluso dentro del rango de tiempo en que ya el Banco Central de la República Dominicana había implementado un esquema de metas de inflación, y utilizan estas relaciones para indagar acerca del nivel de sobre apreciación o sobre depreciación del tipo de cambio dominicano en relación con el dólar estadounidense.

Asimismo, Sánchez-Fung (2005) comprueba que no solo en el ámbito internacional la política monetaria se preocupa por el tipo de cambio, principalmente por su mandato de estabilización de precios, sino también en la República Dominicana, encontrando que el Banco Central este país tiene un sesgo hacia el tipo de cambio en su ejercicio de política monetaria, recalcando que estos hallazgos están en línea con el denominado miedo de dejar flotar el tipo de cambio (fear of floating) discutido por Calvo y Reinhart (2002). Esta condición de la política monetaria, especialmente frecuente en las economías emergentes, motiva a explorar si existen dinámicas diferenciadas en la dinámica del mercado cambiario dominicano antes y después de la implementación de un esquema de metas de inflación.

A pesar de que existe toda la literatura anterior sobre los determinantes y las dinámicas de interacción del tipo de cambio peso-dólar, su relación 
con el tipo de cambio peso-euro, y la relación de ambos con el mercado internacional, no parece haberse tratado formalmente hasta ahora. Esta es la brecha en la literatura que el presente estudio apunta a abordar.

\section{Metodología}

\section{Hipótesis para evaluar}

Este estudio pretende lidiar con cuatro hipótesis principales:

a. Primero se espera que la relación entre el tipo de cambio peso-dólar y peso-euro (efectivamente un tipo de cambio eurodólar implícito) en la República Dominicana es congruente a largo plazo con el tipo de cambio eurodólar en el mercado internacional.

b. Segundo, la relación entre este tipo de cambio eurodólar implícito y el del mercado internacional no se cumple totalmente en el corto plazo, existiendo desviaciones desde su valor esperado.

c. Tercero, el mercado cambiario local en República Dominicana eventualmente se corrige de tal forma que el tipo de cambio eurodólar implícito local retorne a su relación de equilibrio con el internacional.

d. Cuarto, y final, la dinámica anteriormente planteada cambió posterior a la implementación de un esquema de metas de inflación por parte del Banco Central de la República Dominicana.

\section{Estrategia de investigación}

Con el fin de evaluar las hipótesis planteadas anteriormente la metodología propuesta para la elaboración del presente estudio consiste en tres bloques. Primero, se estima un tipo de cambio eurodólar implícito en las tasas de cambio peso/dólar y peso/euro en el mercado cambiario dominicano. Luego, se estima el traspaso desde el tipo de cambio eurodólar oficial internacional al implícito local. Finalmente, se evalúa si la dinámica del traspaso del tipo de cambio internacional al local cambió posterior a la implementación del esquema de metas de inflación por parte del Banco Central de la República Dominicana. 


\section{Estimación del tipo de cambio eurodólar implícito local}

El tipo de cambio eurodólar implícito local en fecha se define para los fines de este estudio como:

$$
\text { Eurodólar Implícito } \text { Local }_{t}=\frac{\text { Tipo de Cambio Peso } / \text { Euro }_{t}}{\text { Tipo de Cambio Peso } / \text { Dólar }}
$$

\section{Cointegración y un modelo de corrección de errores}

Habiendo encontrado en la revisión de la literatura que la presencia de cointegración es un fenómeno que se intenta probar con frecuencia al trabajar investigaciones sobre los mercados de divisas, este trabajo tomaría como primer paso el intento de prueba de esta condición, para luego intentar estimar un modelo de corrección de errores (ECM), según lo propuesto por Engle y Granger (1987), en que se asume que dos series de tiempo están relacionadas en el largo plazo de la forma:

$$
y_{t}=\alpha_{0}+\alpha_{1}^{*} x_{t}+e_{t}
$$

Entonces, se procede a probar si el residuo $e_{t}$ es estacionario, y de probarse se afirma que las series están cointegradas. Luego, se puede estimar una ecuación que se denomina de "corto plazo" de la siguiente forma:

$$
\Delta y_{t}=\beta_{0}+\beta_{1}^{*} \Delta x_{t-1}+\beta_{2} * \Delta y_{t-1}+\beta_{3} * e_{t-1}+v_{t}
$$

De esta forma, se permite que el desvío de la relación de largo plazo entre las dos variables sea incorporado en la ecuación, así modelando la dinámica de retorno hacia ese equilibrio de largo plazo. La velocidad de corrección de la serie a su nivel de largo plazo está entonces dada por $\frac{1}{\beta_{3}}$, expresada en las unidades de tiempo en que se hayan definido las observaciones.

\section{Traspaso del tipo de cambio oficial internacional al implícito local}

La estrategia de estimación propuesta incorpora las hipótesis que se desea probar. Primero, se estima un modelo de largo plazo tal que el 
eurodólar implícito local es determinado por el eurodólar internacional oficial más una constante:

$$
\log \left({\text { Eurodólar Impícito } \left.\text { Local }_{t}\right)=\beta_{0}+\beta_{1} * \log (\text { Eurodólar Internacional }}_{t}\right)+e_{t}
$$

Luego, se estima una ecuación de corto plazo que captura la forma en que el tipo de cambio eurodólar implícito local se corrige a su relación de largo plazo con el internacional, medido a través del coeficiente que acompaña al error de la ecuación de largo plazo $e_{(t-1)}$ con un rezago. Esta ecuación explica la diferencia logarítmica del tipo de cambio eurodólar implícito local a partir de:

- Rezagos de la diferencia logarítmica del eurodólar implícito local.

- Rezagos de la diferencia logarítmica del eurodólar internacional.

- Error de la ecuación de largo plazo rezagado.

Se espera que, si verdaderamente existe una relación de largo plazo, el coeficiente que acompañaría al error de largo plazo en la ecuación de corto plazo sería negativo. Su magnitud indicaría la velocidad de corrección, tal que la unidad entre el coeficiente indica la cantidad de días que tomaría en promedio un desvío de la relación de equilibrio de largo plazo en corregirse.

\section{Selección de la cantidad de rezagos en el modelo de corto plazo}

La selección de la longitud de los rezagos de la diferencia logarítmica del eurodólar implícito local y el internacional se hará según el criterio de información de Akaike (1974), o AIC, que propone el mismo como una forma de tomar en cuenta, al elegir entre varios modelos, conjuntamente el nivel de ajuste del modelo y la simplicidad de este.

\section{Evaluación del impacto de la implementación del esquema de metas de inflación}

Para los fines de evaluar el impacto de la implementación del esquema de metas de inflación realizada por el Banco Central en el año 2012, se propone incluir una variable dicotómica que indique la presencia del 
esquema de meta de inflación, y que esta sea interactuada con el coeficiente de ajuste al largo plazo. Esto constituiría evidencia de la presencia de cambios estructurales en las series de tiempo, manifestándose en la velocidad de corrección de las desviaciones de corto plazo hacia el equilibrio de largo plazo.

\section{Consideraciones sobre la posibilidad del impacto de las comisiones}

Es posible que en el mercado de monedas extranjeras en la República Dominicana existan estructuras de costos, como comisiones, que desincentiven el arbitraje, en el caso de existir el mismo. Dado esto, es posible que las mismas tengan incidencia sobre la dinámica de funcionamiento de este mercado e influyan en la forma en que los mercados se mantienen o salen de equilibrio, y la velocidad en la que retornan al mismo.

Actualmente no se conocen informaciones públicamente disponibles sobre el sistema de comisiones de este mercado, por lo que esta investigación se abstiene de tratar el tema empíricamente. Posteriores trabajos pueden explorar este tema a través de, por ejemplo, una serie de entrevistas a agentes del mercado que determinen la existencia o no de estas estructuras de costos y cómo afectan el funcionamiento del mercado, de existir.

\section{Datos}

\section{Fuentes de información}

Los datos referentes al tipo de cambio venta de ventanilla peso/dólar y peso/euro utilizados para los fines del presente estudio se obtuvieron a partir de las estadísticas oficiales del Banco Central de la República Dominicana. La serie estadística del eurodólar internacional fue descargada desde la Reserva Federal de St. Louis. Se utilizó la ventana de tiempo desde el 2008 al 2018.

Se eligieron fuentes de datos con frecuencia diaria con el interés de capturar las relaciones de corto plazo de los tipos de cambio, donde se espera que verdaderamente ocurran las oportunidades de arbitraje/corrección a la media en un mercado financiero. 


\section{Descripción estadística de los datos}

La Tabla 1 muestra la descripción estadística de las series trabajadas en esta investigación. Se nota que ambos, el tipo de cambio eurodólar implícito local como el internacional, mantienen estadísticos similares, tanto en términos de su primer momento (media y mediana), segundo momento (desviación estándar) y tercer y cuarto momento (asimetría y curtosis). Se evidencia que, por la frecuencia diaria escogida para los fines de esta investigación, se cuenta con una muestra de más de 2,700 observaciones, con valores no disponibles en fines de semana y días feriados para el mercado local e internacional (por esto la disimilitud entre el número de observaciones para cada serie).

Tabla 1. Resumen estadístico de las series de eurodólar

\begin{tabular}{lcc}
\hline & Eurodólar Implícito Local & Eurodólar Internacional \\
\hline Media & 1.30 & 1.28 \\
Mediana & 1.31 & 1.30 \\
Máximo & 1.59 & 1.60 \\
Mínimo & 1.09 & 1.04 \\
Desviación Estándar & 0.12 & 0.13 \\
Asimetría & 0.17 & 0.13 \\
Curtosis & 2.28 & 2.21 \\
Observaciones & 2,739 & 2,757 \\
\hline
\end{tabular}

Nota: se muestra el resumen estadístico de ambas series de eurodólar.

Fuente: cálculos del autor a partir del Banco Central y la Reserva Federal de St. Louis.

\section{Pruebas de la estacionariedad de las series}

Se comprueba mediante la prueba de Dickey Fuller ampliada ${ }^{a}$, cuyos resultados se encuentran debajo en la Tabla 2, que las series son estacionarias solo al ser diferenciadas.

${ }^{\mathrm{a}}$ Ver Dickey y Fuller, (1981). 
Tabla 2. Prueba de estacionariedad de Dickey Fuller ampliada

\begin{tabular}{cccccc}
\hline & & Estadístico & \multicolumn{3}{c}{ Valores Críticos del Estadístico } \\
& & de Prueba & $1 \%$ & $5 \%$ & $10 \%$ \\
\hline Eurodólar & Nivel & -1.25 & -3.43 & -2.86 & -2.57 \\
Implícito Local & 1ra Diferencia & -27.73 & -3.43 & -2.86 & -2.57 \\
\hline Eurodólar & Nivel & -1.91 & -3.43 & -2.86 & -2.57 \\
Internacional & 1ra Diferencia & -49.98 & -3.43 & -2.86 & -2.57 \\
\hline
\end{tabular}

Nota: se muestran el estadístico de la prueba de Dickey Fuller aumentada para las series del eurodólar implícito en el mercado local y el internacional en diferencia y niveles, junto con los valores críticos que definen si se puede rechazar o no la hipótesis nula de la presencia de una raíz unitaria.

Fuente: cálculos del autor.

\section{Evolución de las series en el tiempo}

En la figura 1 se nota tanto la evolución del tipo de cambio eurodólar implícito en el mercado cambiario dominicano como el del mercado internacional. Se observa que ambos mantienen una relación estrecha, pero progresivamente desde 2013 se ha mantenido una brecha significativa entre ellos.

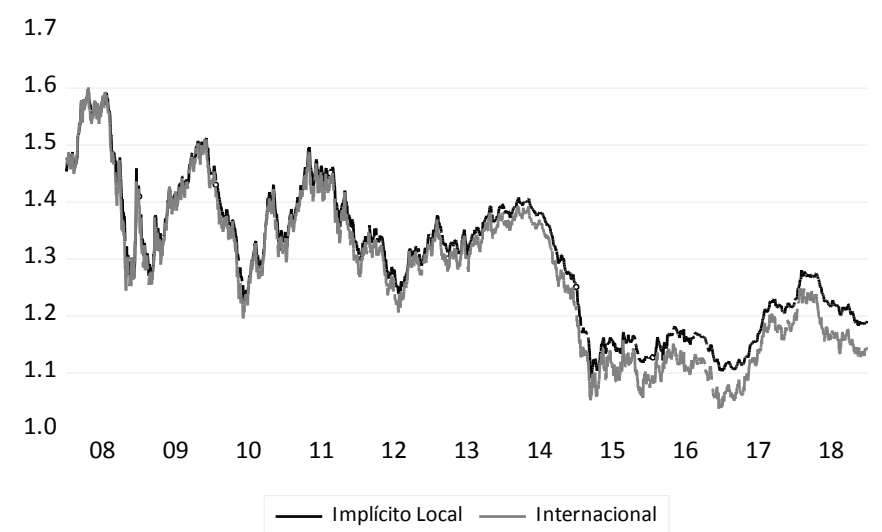

Figura 1. Evolución del tipo de cambio eurodólar

Fuente: el tipo de cambio implícito local corresponde a la división del tipo de cambio venta de ventanilla peso/euro entre el peso/dólar publicados por el Banco Central de la República Dominicana. El tipo de cambio internacional se obtuvo a partir de la Reserva Federal de St. Louis. 


\section{Resultados}

La Tabla 3 muestra los resultados de la estimación de la relación de largo plazo entre el tipo de cambio eurodólar implícito local y el internacional. El resultado indica que por cada $1 \%$ de cambio en el internacional, el implícito local se mueve en $0.886 \%$ en la misma dirección previo a la implementación del esquema de metas de inflación; posterior al mismo, el traspaso aumenta en $0.008 \%$.

TABla 3. ECUACiÓN DEL EURODÓLAR IMPLÍ́CITO LOCAL EN ECUACIÓN DE LARGO PLAZO

\begin{tabular}{lcccc}
\hline \multicolumn{1}{c}{ Variable } & \multirow{2}{*}{ Coeficiente } & Error & Estadístico de & Valor \\
& & Estándar & Prueba T & Probabilístico \\
\hline Log(USD/EUR) & 0.886 & 0.003 & 261.079 & 0.000 \\
Log(USD/EUR)*d_emi & 0.008 & 0.003 & 2.531 & 0.011 \\
Constante & 0.048 & 0.001 & 48.093 & 0.000 \\
\hline R2 $99.14 \%$ & & Observaciones 2,536 \\
\multicolumn{2}{c}{ R2 Ajustado 99.14\% } & & & \\
\hline
\end{tabular}

Nota: se muestran los resultados de la estimación de la ecuación de largo plazo descrita en la sección de metodología.

Fuente: cálculos del autor.

Se observa a través de la prueba de Engle y Granger ${ }^{\mathrm{b}}$ mostrada en la Tabla 4 que las series cointegran en el largo plazo, lo que permite hacer estimar este tipo de relaciones entre series integradas de orden uno (1) sin temer la presencia de correlación espuria.

\section{Tabla 4. Prueba de cointegración de Engle y Granger}

\begin{tabular}{ccc}
\hline & Estadístico de Prueba & Valor Probabilístico \\
\hline Engle-Granger tau-statistic & -15.10 & 0.00 \\
Engle-Granger z-statistic & -425.13 & 0.00 \\
\hline
\end{tabular}

Nota: se muestran los resultados de la prueba de Engle y Granger para las series del tipo de cambio eurodólar implícito local y el internacional. La prueba evalúa si es posible rechazar la hipótesis nula de no cointegración entre las series. El valor probabilístico se estima según la metodología propuesta en MacKinnon (1996).

Fuente: cálculos del autor.

bVer Engle y Granger (1987). 
Luego de haber comprobado que sí existe una relación de cointegración entre el eurodólar implícito local y el internacional, se procede a estimar una batería de modelos para la ecuación de corto plazo y compararlos entre sí para probar cuál es la especificación con mejor ajuste. Se eligió no probar más de cinco días de rezago en cada variable porque toda la literatura internacional consultada indica que la dinámica de este mercado es de alta velocidad de reacción, notándose que en muchos casos el mercado de divisas reacciona incluso hasta en minutos a informaciones nuevas. En la Tabla 5 se muestran los resultados de esta prueba, emergiendo el modelo con cuatro rezagos del tipo de cambio eurodólar implícito local y tres del internacional como el de mejor ajuste estadístico según la prueba utilizada (AIC).

Tabla 5. Criterio AiC resultante de batería de modelos probados

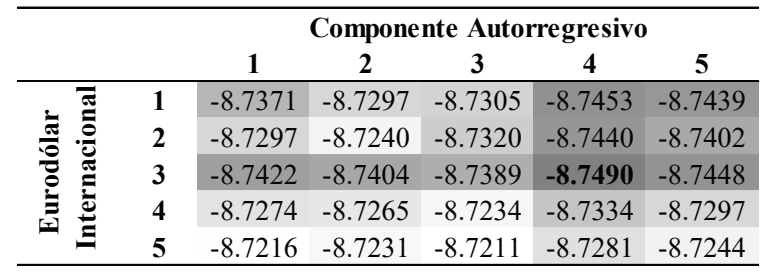

Nota: se muestran el criterio AIC ${ }^{c}$ de la batería de 25 modelos probados con distintas combinaciones de rezagos tanto de la variable explicada (eurodólar implícito local) como del internacional en la ecuación de corto plazo. La escala de grises índica visualmente el valor de cada número (dirección negativa), y se señala el resultado con el menor AIC, indicando el mejor ajuste.

Fuente: realizados por el autor.

Habiendo definido la especificación con mejor ajuste, se muestran los resultados de esta en la Tabla 6 . Se comprueba la existencia de un componente autorregresivo, evidenciado en la significancia conjunta de cuatro rezagos de la diferencia logarítmica del tipo de cambio eurodólar implícito local (USD_EUR_I). También el impacto del tipo de cambio eurodólar internacional (USD_EUR) resulta estadísticamente significativo, con un signo positivo.

'Ver Akaike (1974). 
Se comprueba en la Tabla 6 primero que en el corto plazo el eurodólar implícito local se corrige hacia su nivel de equilibrio de largo plazo, pero la velocidad de traspaso cambió radicalmente posterior a la implementación del esquema de metas de inflación. Previo al esquema, el coeficiente del traspaso era de -0.194 , equivalente a una corrección al largo plazo que tomaba 5.15 días $\left(\frac{1}{0.194}\right)$. Posterior a la implementación del esquema de metas de inflación, esta velocidad de traspaso pasó a 19.6 días $\left(\frac{1}{0.1940-.143}\right)$, casi cuadriplicando tiempo de corrección. Esto significa que posterior al 2012, el tipo de cambio eurodólar local toma cuatro veces más tiempo en promedio para retornar al nivel de equilibrio de largo plazo que sostiene con el eurodólar internacional. Estos hallazgos son consistentes con la literatura internacional, que muestra, con el interés de mantener su mandato de estabilización de precios, los bancos centrales (especialmente de economías emergentes) tienen cierta injerencia en los mercados cambiarios y crean desequilibrios temporales en los precios de las divisas.

Tabla 6. ECUACIÓN DEL EUROdÓLAR IMPLÍCITO LOCAL EN ECUACIÓN DE LARGO PLAZO

\begin{tabular}{|c|c|c|c|c|}
\hline Variable & Coeficiente & $\begin{array}{c}\text { Error } \\
\text { Estándar }\end{array}$ & $\begin{array}{c}\text { Estadístico de } \\
\text { Prueba T } \\
\end{array}$ & $\begin{array}{c}\text { Valor } \\
\text { Probabilístico } \\
\end{array}$ \\
\hline Constante & 0.000 & 0.000 & -0.411 & 0.681 \\
\hline DLOG(USD_EUR_I(-1)) & -0.161 & 0.026 & -6.131 & 0.000 \\
\hline DLOG(USD_EUR_I(-2)) & -0.094 & 0.025 & -3.714 & 0.000 \\
\hline DLOG(USD_EUR_I(-3)) & 0.037 & 0.022 & 1.683 & 0.093 \\
\hline DLOG(USD_EUR_I(-4)) & 0.045 & 0.021 & 2.175 & 0.030 \\
\hline DLOG(USD_EUR(-1)) & 0.224 & 0.016 & 13.950 & 0.000 \\
\hline DLOG(USD_EUR(-2)) & 0.127 & 0.017 & 7.624 & 0.000 \\
\hline DLOG(USD_EUR(-3)) & 0.083 & 0.015 & 5.413 & 0.000 \\
\hline ECM_V(-1) & -0.194 & 0.015 & -13.151 & 0.000 \\
\hline ECM_V(-1)*D_EMI & 0.143 & 0.017 & 8.597 & 0.000 \\
\hline \multicolumn{2}{|c|}{ R2 $35.20 \%$} & \multicolumn{3}{|c|}{ Observaciones 1,897} \\
\hline
\end{tabular}

Nota: se muestran los resultados de la estimación de la ecuación de largo plazo descrita en la sección de metodología.

Fuente: cálculos del autor. 


\section{Conclusiones}

Los resultados de este estudio apuntan a la comprobación de cuatro ideas principales. Primero, existe una relación de equilibrio entre la razón del tipo de cambio local peso/euro y peso dólar, efectivamente una tasa eurodólar implícita y el eurodólar oficial internacional. Segundo, existen momentos en que ocurren desviaciones de esta relación de equilibrio, y aquí yacen aún oportunidades de arbitraje en el mercado financiero local de República Dominicana. Tercero, la velocidad de corrección de estos desequilibrios es relativamente rápida, siendo siempre esta menor a un mes. Cuarto, la implementación del esquema de metas de inflación por parte del Banco Central de la República Dominicana parece haber introducido rigideces al mercado cambiario local, lo que se ha reflejado en la desaceleración de esta velocidad de ajuste, que pasó de cinco días a alrededor de veinte.

Futuros estudios pueden ampliar esta rama de investigación en República Dominicana abordando el tratamiento de temas como el posible impacto de las comisiones en el mercado de divisas, el tratamiento de los residuos del modelo de corto plazo a través de una metodología del tipo $\mathrm{ARCH}^{\mathrm{d}}$ que modele la volatilidad de los residuos y permita realizar predicciones de esta varianza, la interacción del tipo de cambio peso-dólar y peso-euro con las otras razonas cambiarias que se manejan en República Dominicana, el impacto de las desviaciones del equilibrio de largo plazo del eurodólar implícito (encontradas en este trabajo) en las variables macroeconómicas y financieras del país, entre otras posibles líneas de investigación.

Finalmente, parecería que queda pendiente una línea de indagación sobre la razón por la que las acciones de los agentes del mercado financiero dominicano no reducen la velocidad de ajuste de la paridad implícita del eurodólar, ya que el ejercicio del arbitraje debería tender a tener exactamente este efecto. Un punto de partida para este análisis pueden ser trabajos como Gromb y Vayanos (2010), quienes reúnen una amplia literatura que incorpora diversas razones (riesgo no fundamental, costos

${ }^{\mathrm{d}}$ Modelos autorregresivos con heterocedasticidad condicional (ARCH, por sus siglas en inglés). Ver Engle (1982). 
de tomar la posición corta o larga, limitaciones al apalancamiento, limitaciones de capital disponible, entre otras) para construir modelos que reflejan la realidad de que en algunas instancias existen condiciones de arbitraje en un mercado que tienden a no desaparecer tan rápido como la teoría de los mercados eficientes haría pensar.

\section{Referencias}

Akaike, H. (1974). A new look at the statistical model identification. IEEE Transactions on Automatic Control, 19(6), 716-723. doi:10.1109/ TAC.1974.1100705

Akram, Q., Rime, D. y Sarno, L. (2008). Arbitrage in the foreign exchange market: turning on the microscope. Journal of International Economics, 76, 237-253. doi: 10.1016/j.jinteco.2008.07.004

Andújar, J. y Medina, A. (2008). Un modelo macroeconómico de pequeña escala para la República Dominicana. En: Modelos macroeconométricos de la banca central: Centroamérica y República Dominicana. México: Comisión Económica para América Latina y el Caribe, 179-219.

Baillie, R. T. y Bollerslev, T. (1989). Common Stochastic Trends in a System of Exchange Rates. The Journal of Finance, 44(1), 167-181. doi: 10.1111/j.1540-6261.1989.tb02410.x

Baillie, R. T. y Bollerslev, T. (1994). Cointegration, Fractional Cointegration, and Exchange Rate Dynamics. The Journal of Finance, 49(2), 737-745. doi: $10.2307 / 2329172$

Baillie, R. y Osterberg, W. (2000). Deviations from daily uncovered interest rate parity and the role of intervention. Journal of International Financial Markets, Institutions and Money, 10(3-4), 363-379. doi: 10.1016/S1042-4431(00)00029-9

Bello, O., Heresi, R. y Pineda, R. (2010). El tipo de cambio real de equilibrio: un estudio para 17 paises de América Latina. Santiago de Chile, Chile: Serie Macroeconómica del Desarrollo, 82. CEPAL ISBN: 9789213232910

Calvo, G. A. y Reinhart, C. (2002). Fear of floating. Quarterly Journal of Economics, 117(2), 379-408. doi: 10.3386/w7993 
Chinn, M., y Meredith, G. (2004). Monetary Policy and Long Horizon Uncovered Interest Parity. IMF Staff Papers 51(3), 409-430.

Copeland, L. S. (1991). Cointegration Tests with Daily Exchange Rate Data. Oxford Bulletin of Economics and Statistics, 53(2), 185-198. doi: 10.1111/j.1468-0084.1991.mp53002005.x

Cruz, A. (2016). Tipo de cambio real en la República Dominicana: enfoques alternativos de equilibrio y desalineamiento. Ciencia y Sociedad, 41(3), 589-616. doi: 10.22206/CYS.2016.V41I3.PP589-616

Dickey, D. y Fuller W. (1981). Likelihood ratio statistics for autoregressive time series with a unit root. Econometrica, 49(4):1057-1077. doi: $10.2307 / 1912517$

Diebold, F. X., Gardeazabal, J., y Yilmaz, K. (1994). On cointegration and exchange rate dynamics. The Journal of Finance, 49(2), 727-735. doi: 10.1111/j.1540-6261.1994.tb05160.x

Engle, R. F. (1982). Autoregressive Conditional Heteroscedasticity with Estimates of the Variance of United Kingdom Inflation. Econometrica, 50(4), 987-1007. doi: 10.2307/1912773

Engle, R. F. y Granger C. W. J. (1987). Cointegration and error correction: Representation, estimation and testing. Econometrica, 55(2): 251-276. doi: 10.2307/1913236

Fama, E. F. (1970). Efficient Capital Markets: A Review of Theory and Empirical Work. The Journal of Finance, 25(2), 383-417. doi: $10.2307 / 2325486$

Granger, C. W. J. y Newbold, P. (1974) Spurious Regressions in Econometrics. Journal of Econometrics, 2(2), 111-120. doi: 10.1016/03044076(74)90034-7

Granger, C. W. J. (1986). Developments in the Study of Cointegrated Economic Variables. Oxford Bulletin of Economics and Statistics, 48(3), 213-228. doi: 10.1111/j.1468-0084.1986.mp48003002.x

Gratereaux, C. M. (2015). Tipo de cambio real de equilibrio, fundamentos y desalineamiento en una economía pequeña y abierta: Metodología BEER adaptada al caso dominicano. Nueva Literatura Económica Dominicana, 19. Banco Central de la República Dominicana.

Gromb, D. y Vayanos, D. (2010). Limits of Arbitrage: The State of the Theory. Annual Review of Financial Economics, 2, 251-275. doi: 10.1146/annurev-financial-073009-104107 
Grossman, S.J. y Stiglitz, J.E. (1976). Information and competitive price systems. American Economic Review, 66(2), 246-253.

Grossman, S.J., y Stiglitz, J.E. (1980). On the impossibility of informationally efficient markets. American Economic Review, 70, 393-408.

Haug, A. A., MacKinnon, J. G. y Michelis, L. (2000). European Monetary Union: A Cointegration Analysis. Journal of International Money and Finance, 19(3), 419-432. doi: 10.1016/S0261-5606(00)00013-9

Lajaunie, J. P. y Naka, A. (1997). Re-Examining Cointegration, Unit Roots and Efficiency in Foreign Exchange Rates. Journal of Business Finance \& Accounting, 24(3), 363-374. doi: 10.1111/1468-5957.00109

Mark, N. C. y Moh, Y. K. (2007). Official interventions and the forward premium anomaly. Journal of Empirical Finance, 14(4), 499-522. doi: 10.1016/j.jempfin.2006.10.002

McCallum, B. (1994). A Reconsideration of the Uncovered Interest Rate Parity Relationship. Journal of Monetary Economics 33(1), 105-132. doi: 10.1016/0304-3932(94)90016-7

MacKinnnon, J. (1996). Numerical distribution functions for unit root and cointegration tests. Journal of Applied Econometrics, 11(6):601-618. doi: 10.1002/(SICI)1099-1255(199611)11:6\%3C601::AID-JAE417\%3E3.0.CO;2-T

Norrbin, S. C. (1996). Bivariate Cointegration among European Monetary System Exchange Rates. Applied Economics, 28(12), 1505-1513. doi: 10.1080/000368496327499

Phengpis, C. (2006). Market efficiency and cointegration of spot exchange rates during periods of economic turmoil: Another look at European and Asian currency crises. Journal of Economics and Business, 58(4), 323,342. doi: 10.1016/j.jeconbus.2005.10.003

Rashid, S. (2007). The Law of One Price: Implausible, Yet Consequential. The Quarterly Journal of Austrian Economics, 10(1), 79-90. doi: 10.1007/s12113-007-9001-7

Sánchez-Fung, J. R. (1999). Efficiency of the black market for foreign exchange and PPP: the case of the Dominican Republic. Applied Economics Letters, 6(3), 173-176. doi: 10.1080/135048599353573

Sánchez-Fung, J. R. (2005). Estimating a monetary policy reaction function for the Dominican Republic. International Economic Journal, 19(4), 563-577. doi: 10.1080/10168730500382121 
Sánchez-Fung, J. R. (2014). Exchange rate dynamics, forecasting and the microstructure approach: empirical evidence for an emerging market economy. Macroeconomics and Finance in Emerging Market Economies, 8(1-2), 81-89. doi: 10.1080/17520843.2014.977929

Sephton, P. S. y Larsen, H. K. (1991). Tests of Exchange Market Efficiency: Fragile Evidence from Cointegration Tests. Journal of International Money and Finance, 10(4), 561-570. doi: 10.1016/02615606(91)90007-7

Vásquez-Ruíz, H. \& Rivas, R. A. (2012). Estimación del tipo de cambio real de equilibrio en la República Dominicana. Serie de Estudios Económicos, 8. Banco Central de la República Dominicana.

Vásquez-Ruíz, H. \& Rivas, R. A. (2014). Análisis del tipo de cambio real en la República Dominicana: Un estudio con base en metodologías de estimación del Fondo Monetario Internacional. Monetaria, 36(1), 145-176.

Woo, K. Y. (1999). Cointegration Analysis of the Intensity of the ERM Currencies under the European Monetary System. Journal of International Financial Markets, Institutions and Money, 9(4), 393-405. doi: 10.1016/S1042-4431(99)00016-5 